\title{
Gliptin repurposing for COVID-19
}

2

Angel Jonathan Ruiz Moreno, ${ }^{1,2,5}$ Atilio Reyes Romero, ${ }^{1}$ Constantinos Neochoritis, ${ }^{3,6}$ Matthew Groves, ${ }^{1}$ Marco Velasco-Velázquez, ${ }^{2,5}$ Alexander Dömling ${ }^{1,3,4 *}$

${ }^{1}$ Department of Drug Design, University of Groningen, The Netherlands

${ }^{2}$ Departamento de Farmacología y Unidad Periférica de Investigación en Biomedicina Traslacional, Facultad de Medicina, Universidad Nacional Autónoma de México (UNAM). Ciudad de México, México.

${ }^{3}$ Telesis Pharma, Groningen, The Netherlands

${ }^{4}$ FORTH, Institute of Molecular Biology and Biotechnology, Crete, Greece

${ }^{5}$ Programa de Doctorado en Ciencias Biomédicas, UNAM. Ciudad de México, México.

${ }^{6}$ University of Crete, Department of Chemistry, Greece.

\section{Background}

De novo discovery of any therapeutic (e.g. antibodies, vaccines or small molecules) takes years to move from idea/preclinic to market release and is not a short-term solution for the current SARS-CoV-2 pandemic. Therefore, drug repurposing - the discovery of novel indication areas for already approved drugs is perhaps the only approach able to yield a short-term solution.

\section{Methods}

In order to repurpose drugs as potential inhibitors of SARS-CoV-2 proteases 3CLpro and PLpro, we performed a computational screening using the oral bioavailable antidiabetic drug class of gliptins as candidates.

\section{Results}

The orally bioavailable antidiabetic drug class of gliptins is safe and has been clinically available (and used by millions of patients) since 2006. Based on our repurposing hypothesis and our computational docking we predict the nitrile containing gliptins to inhibit the SARS-CoV-2 proteases 3CLpro and PLpro. As a result, nitrile containing gliptins deserve further investigation as potential anti-COVID19 drugs.

In December 2019, a novel coronavirus (SARS-CoV-2 the cause of the disease named COVID-19) was found in Wuhan, China. ${ }^{1}$ Human-to-human transmission has occurred among close contacts. ${ }^{2}$ As of 30 March 2020, there have been over 700.000 reported cases with over 30.000 deaths for the SARS-CoV-2 pandemic worldwide. However, there are currently no effective medications against SARS-CoV-2.

De novo drug discovery historically takes years from idea/preclinic to the market, independently of the nature of the drug (e.g. small molecule, antibody, vaccine). Thus, this strategy is not a short-term solution for the current pandemic. Drug repurposing aims to discover novel indications for already approved 
drugs. ${ }^{3}$ The overwhelming advantage of drug repurposing is the much faster clinical approval, due to already extensive knowledge of the physicochemical properties and behavior of the drug in humans. Therefore, many groups have pursued a drug repurposing approach. This has led to several promising drugs which are currently tested for COVID-19 in humans, including Favipiravir, Remdesivir, HIV protease inhibitors, chloroquine or Tozilizumab. ${ }^{4,5}$ So far, however, none showed more than promising activity based on low numbers of patients per trial. Additionally, a combination of Lopinavir and Ritonavir (Kaletra ${ }^{\circledR}$ ) - effective for HIV infection - failed in 200 severe COVID-19 cases in China. ${ }^{5}$ Lopinavir and Ritonavir did not show any benefit over the current standard therapy. Clearly, additional drug repurposing hypotheses need to be tested.

In our search for drugs potentially inhibiting the replication of the SARS-CoV-2 we concentrated on compounds inhibiting the proteases. Both the virus encoded proteases, including the $3 \mathrm{C}$-like proteinase (3CLpro) and Papain-like proteinase (PLpro), are highly conserved among CoVs and are involved in the essential processing of the viral polyprotein in a coordinated manner. Therefore, they represent important drug targets. 3 CLpro cleaves the C-terminus of replicase polyprotein at 11 sites. ${ }^{6}$ Recently, the Å resolution $3 \mathrm{D}$ X-ray structure was reported of the 3 CLpro covalently bound to a peptidomimetic acrylester (PDB ID 6LU7). ${ }^{7}$ PLpro is responsible for the cleavages located at the $\mathrm{N}$-terminus of the replicase polyprotein and has other important functions during the viral replication cycle. ${ }^{8}$ Thus, we concentrated our investigations on drug classes with a reactive warhead potentially reacting with the 3CLpro active site Cys145 and/or PLpro active site Cys112, respectively.

Gliptins are a class of oral hypoglycemics that inhibit the enzyme dipeptidyl peptidase-4 (DPP-4), a serine protease that rapidly inactivates incretin hormones in plasma. They are widely used as drugs to treat diabetes mellitus type 2 . The first compound - sitagliptin - received market approval in 2006. Since then many new gliptins were introduced (Fig. 1A, B). Mechanistically, gliptins can be divided in two classes, covalent and non-covalent inhibitors. ${ }^{9}$ The nitrile containing gliptins make a covalent bond with the active site Ser630 of DPP-4 (Fig. 1C). Nitrile containing gliptins include Saxapliptin, Vildagliptin, Bisegliptin, Trelagliptin, Anagliptin, Melogliptin, and Denagliptin (Fig.1B). We hypothesized that nitrile containing gliptins might be potential repurposing candidates given that cysteines are well known to react with nitriles to form covalent adducts in a similar way as serines (Fig. 1D). For example arylnitriles have been described as cysteine protease inhibitors. ${ }^{11}$ To form stable adducts the covalent imidate is often stabilized by a hydrogen bonding network to the receptor. In the case of Vildagliptin, the imidate NH forms hydrogen bondings to the Tyr547 $\mathrm{OH}$ and an adjacent water molecule (Figure 1C). 

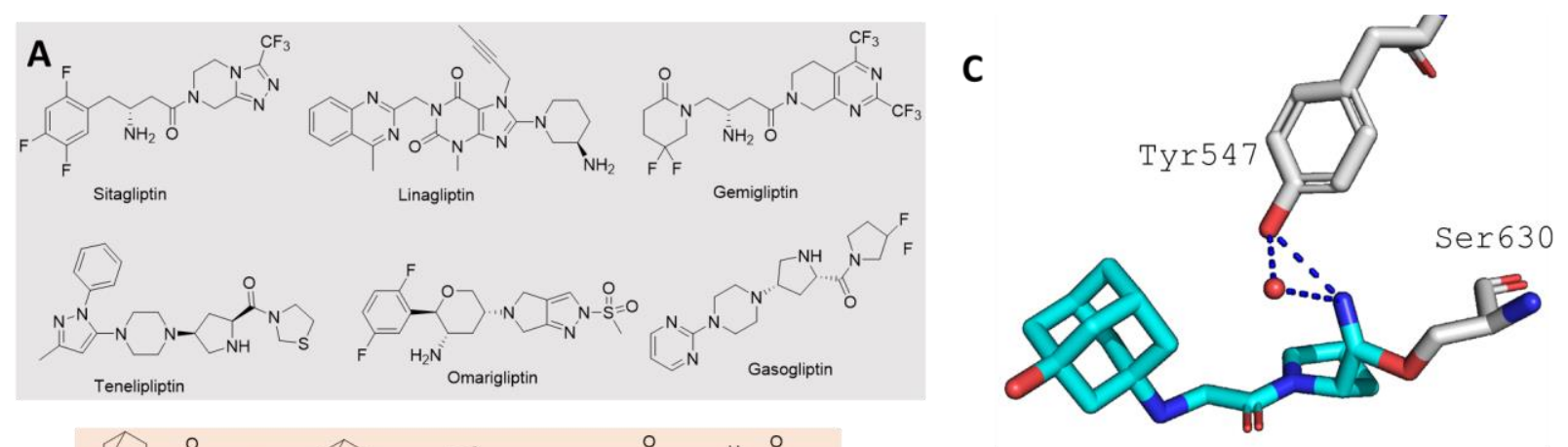

B

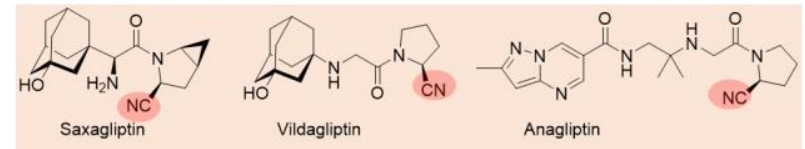

D

67

Figure 1. Antidiabetic gliptins as potential COVID-19 repurposing drugs. A) Marketed or late stage noncovalent DPP-4 inhibitors. B) Marketed or late stage covalent DPP-4 inhibitors with electrophile nitrile circled in red. C) Example (PDB ID 6B1E) of Vildagliptin (cyan sticks) covalently bound to 3CLpro Ser630 (grey sticks) and the imine group stabilized by a hydrogen bond to Tyr547 (grey sticks) and a water molecule (red ball). ${ }^{10}$ D) Mechanistic similarity of nucleophilic serine and cysteine addition to an electrophilic nitrile.

Methods In order to evaluate the potential of the nitrile containing gliptins as 3CLpro and PLpro inhibitors, we performed covalent docking using GOLD and CovDock software. The potential implication of gliptins binding to the were analyzed by minimizing the complexes comprising best docking poses using the MAB force field (detailed methods in SI). ${ }^{12-14}$ For SARS-CoV-2 3CLpro (PDB ID 6LU7), the catalytic Cys145 was used as docking site. For SARS-CoV-2 PLpro there is no crystal structure yet available. However, the PLpro from SARS-CoV-2 shows an high sequence identity (83\%) with PLpro from SARS-CoV (Fig. S1). ${ }^{15}$ Specifically, in the near vicinity of the active site and binding site of inhibitors they are identical. Thus, we decided to use SARS-CoV PLpro (PDB ID 2FE8) Cys112 as binding site for our docking experiments. Additionally, the cooperative interaction networks were evaluated for the best poses from GOLD and CovDock docking using Scorpion software. ${ }^{3}$

Results and discussion Docking scores and cooperative binding network analysis showed higher values for all gliptins against 3CLpro (Tables S2 and Fig. S2B), suggesting a higher affinity for the gliptins rather than PLpro (Table S1 and Fig. S2A). Vildagliptin was identified as the best dual action candidate for both 3CLpro and PLpro (Fig. 2A, B). On the other hand, Denagliptin and Anaglitin were identified as the best candidates for 3 CLpro and PLpro, respectively. Saxagliptin and secondly Trelagliptin, showed a consensus score value higher against 3CLpro rather than PLpro, indicating a possible higher selectivity for 3CLpro. 
However, Melogliptin and Bisegliptin displayed higher consensus score values for PLpro suggesting a possible selectivity for this target. Finally, as stated previously, scoring values for 3CLpro were higher, indicating that selectivity of gliptins against 3CLpro and more importantly against PLpro must be considered carefully.

Docking results showed that by forming the covalent complex with 3CLpro the best compounds (Vildagliptin, Anagliptin, and Denagliptin) were able to form an extended hydrogen bonding network and/or hydrophobic interaction with the His41 (Fig. 2A). Both, Cys145 and His41 are part of the catalytic dyad of different CoV's main proteases. ${ }^{5}$ Additionally, the scores of Vildagliptin, Anagliptin and Denagliptin and His41 were preserved and in some cases enhanced, suggesting that binding of these gliptins could induce and adjust the fit which potentiates the binding and stabilization of these compound into the catalytic site of 3CLpro (Table S4). Therefore, these gliptins could have a direct impact on the protease $3 C L$ pro function, and consequently impact the replication cycle of SARS-CoV-2.

The protease activity of PLpro has been reported to be catalyzed by the dyad Cys112 and His 273 or the triad additionally involving Asp287. ${ }^{8,15}$ Experimental non-covalent inhibitors of SARS-CoV also have been reported. ${ }^{16,17}$ Interestingly, such inhibitors bind in a cavity next to the catalytic triad but none of them interacts directly with the residues of the catalytic triad, despite proving high potency and selectivity in vitro. ${ }^{16}$ All gliptins are predicted to potentially form covalent adducts to SARS-CoV and SARS-CoV-2 proteases through direct interaction with the Cys112 in the catalytic triad where also other reported inhibitors bind (Fig. S5). Amongst the best candidates for PLpro (Anagliptin, Vildaglipti and Melogliptin) (Fig. 2B, Table S3) only Anagliptin is able to form a hydrogen bonding interaction with the His 273. However, the three proposed gliptins were able to form an extended network of ligand - receptor interactions, mainly hydrogen bonds and hydrophobic interactions, with the same residues as the reported inhibitors: Gly164, Asp165, and Tyr269. Therefore, our results suggest the formation a stable binding of the nitrile containing gliptins (Fig. S3, S4) and a possible inhibition of PLpro activity. 

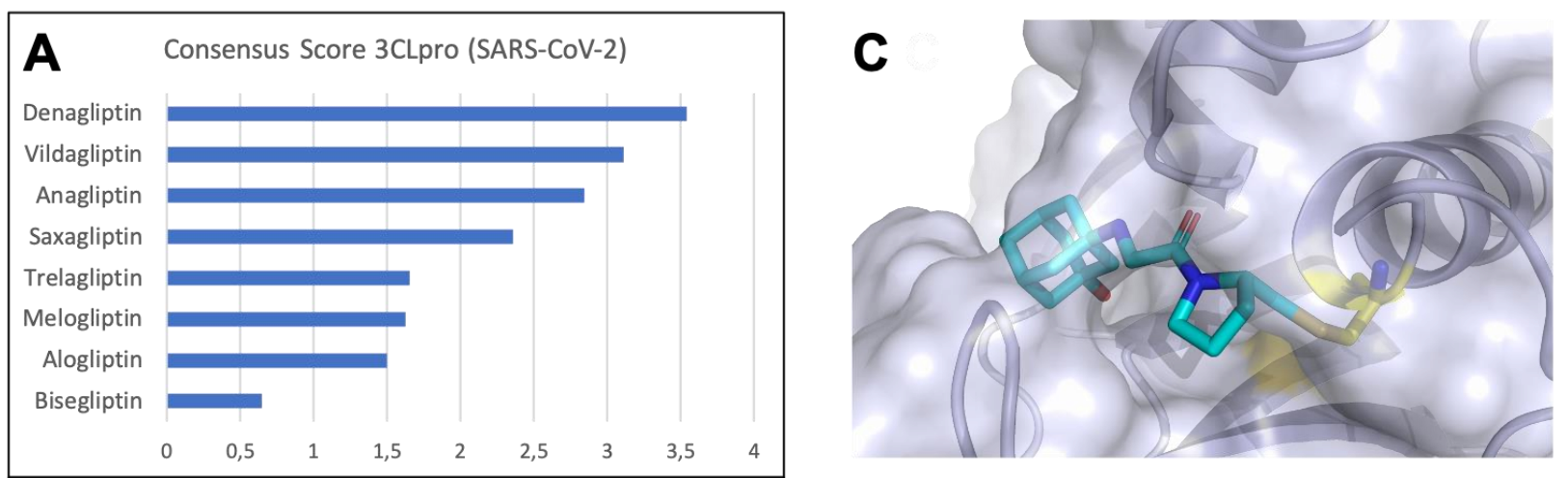

B Consensus Score PLpro (SARS-CoV)
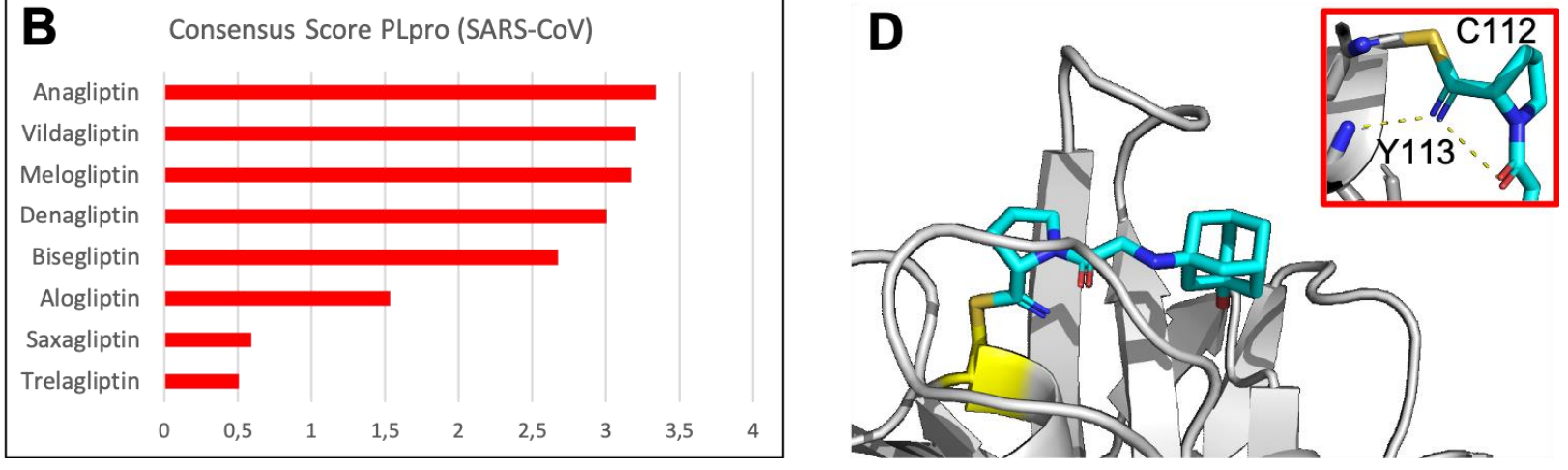

Figure 2. Covalent docking of the nitrile-gliptins into the SARS-CoV-2 protease 3CLpro (PDB ID 6LU7) and SARS-CoV-1 protease PLpro (PDB ID 2FE8). A, B) Consensus docking scores for the nitrile-gliptins for the two proteases. B, C) Highest scoring poses of the best dual action Vildagliptin in 3CLpro and PLpro with some indicated molecular interactions. The active site cysteines forming the covalent bond are shown in yellow. Insert in D) shows the stabilization of the thioimidate structure through an intramolecular hydrogen bond to the pyrrolidine acyl carbonyl and a $\mathrm{H}$-bond donor pi interaction to the backbone $\mathrm{NH}$ of Tyr113.

Gliptin drug safety has been monitored over more than a decade and good tolerance/safety profile of DPP-4 inhibitors has been largely confirmed, importantly including elderly populations. ${ }^{18}$ Gliptins are orally bioavailable, easy to formulate, and can be and are already delivered to millions of people in the form of a pill. They are already manufactured on scale, which is critical in the current pandemic. They are stable at ambient temperature and have a long shelf life. Furthermore, PK/PD is also well established. For example, the plasma half-life of Saxagliptin $(\sim 27 \mathrm{~h})$ is much longer than that of Vildagliptin $(\sim 2 \mathrm{~h})$ and thus Saxagliptin requires lower therapeutic dosage than Vildagliptin: Saxagliptin is administrated $5 \mathrm{mg}$ once a day, while Vildagliptin is $50 \mathrm{mg}$ twice a day. ${ }^{20-22}$ However nothing is known about PK/PD profile of the different gliptins in the lungs. Based on the hypothetical inhibition mechanism of the viral replication through inhibition of the two essential SARS-CoV-2 proteases and the well-established safety profile a preventative and/or curative application of the selected gliptins is thinkable.

Unfortunately, due to the general shutdown of research and academic laboratories worldwide further studies including protease assays and cell-based virus proliferation assays cannot be performed at this time. The severity of the SARS-CoV-2 pandemic prompted us to publish our preliminary results. We hope that our drug repurposing hypothesis could become the starting point to perform further studies in severely ill COVID-19 patients. 


\section{References}

141 1. Chen N, Zhou M, Dong X, et al. Epidemiological and clinical characteristics of 99 cases of 2019 142 novel coronavirus pneumonia in Wuhan, China: a descriptive study. Lancet Lond Engl 143 2020;395(10223):507-13.

$1442 . \quad$ Li Q, Guan X, Wu P, et al. Early Transmission Dynamics in Wuhan, China, of Novel Coronavirus145 Infected Pneumonia. N Engl J Med 2020;382(13):1199-207.

146 3. Pushpakom S, lorio F, Eyers PA, et al. Drug repurposing: progress, challenges and 147 recommendations. Nat Rev Drug Discov 2019;18(1):41-58.

148 4. Harrison C. Coronavirus puts drug repurposing on the fast track. Nat Biotechnol 2020;

$1495 . \quad$ Cao B, Wang Y, Wen D, et al. A Trial of Lopinavir-Ritonavir in Adults Hospitalized with Severe Covid150 19. N Engl J Med 2020;

151 6. Pillaiyar T, Manickam M, Namasivayam V, Hayashi Y, Jung S-H. An Overview of Severe Acute 152 Respiratory Syndrome-Coronavirus (SARS-CoV) 3CL Protease Inhibitors: Peptidomimetics and Small 153 Molecule Chemotherapy. J Med Chem 2016;59(14):6595-628.

$1547 . \quad$ Jin Z, Du X, Xu Y, et al. Structure of Mpro from COVID-19 virus and discovery of its inhibitors. 155 bioRxiv 2020;2020.02.26.964882.

156 8. Báez-Santos YM, St John SE, Mesecar AD. The SARS-coronavirus papain-like protease: structure, 157 function and inhibition by designed antiviral compounds. Antiviral Res 2015;115:21-38.

158 9. Nabeno $\mathrm{M}$, Akahoshi F, Kishida $\mathrm{H}$, et al. A comparative study of the binding modes of recently 159 launched dipeptidyl peptidase IV inhibitors in the active site. Biochem Biophys Res Commun 160 2013;434(2):191-6.

161 10. Berger JP, SinhaRoy R, Pocai A, et al. A comparative study of the binding properties, dipeptidyl 162 peptidase-4 (DPP-4) inhibitory activity and glucose-lowering efficacy of the DPP-4 inhibitors alogliptin, 163 linagliptin, saxagliptin, sitagliptin and vildagliptin in mice. Endocrinol Diabetes Metab 2018;1(1):e00002.

164 11. Ehmke V, Quinsaat JEQ, Rivera-Fuentes $P$, et al. Tuning and predicting biological affinity: aryl 165 nitriles as cysteine protease inhibitors. Org Biomol Chem 2012;10(30):5764-8.

166 12. Jones G, Willett P, Glen RC, Leach AR, Taylor R. Development and validation of a genetic algorithm 167 for flexible docking. J Mol Biol 1997;267(3):727-48.

168 13. Zhu K, Borrelli KW, Greenwood JR, et al. Docking covalent inhibitors: a parameter free approach 169 to pose prediction and scoring. J Chem Inf Model 2014;54(7):1932-40.

170 14. Gerber PR, Müller K. MAB, a generally applicable molecular force field for structure modelling in 171 medicinal chemistry. J Comput Aided Mol Des 1995;9(3):251-68.

172 15. Ratia K, Saikatendu KS, Santarsiero BD, et al. Severe acute respiratory syndrome coronavirus papain173 like protease: Structure of a viral deubiquitinating enzyme. Proc Natl Acad Sci 2006;103(15):5717-22. 
174 16. Báez-Santos YM, Barraza SJ, Wilson MW, et al. X-ray Structural and Biological Evaluation of a 175 Series of Potent and Highly Selective Inhibitors of Human Coronavirus Papain-like Proteases. J Med Chem 176 2014;57(6):2393-412.

177 17. Ratia K, Pegan S, Takayama J, et al. A noncovalent class of papain-like protease/deubiquitinase 178 inhibitors blocks SARS virus replication. Proc Natl Acad Sci 2008;105(42):16119-24.

179 18. Scheen AJ. The safety of gliptins : updated data in 2018. Expert Opin Drug Saf 2018;17(4):387180405.

181 19. He H, Tran P, Yin H, et al. Absorption, metabolism, and excretion of [14C]vildagliptin, a novel 182 dipeptidyl peptidase 4 inhibitor, in humans. Drug Metab Dispos Biol Fate Chem 2009;37(3):536-44.

183 20. Tahrani AA, Piya MK, Barnett AH. Saxagliptin: a new DPP-4 inhibitor for the treatment of type 2 184 diabetes mellitus. Adv Ther 2009;26(3):249-62.

185 21. Dhillon S. Saxagliptin: A Review in Type 2 Diabetes. Drugs 2015;75(15):1783-96.

186 
Angel Jonathan Ruiz Moreno, ${ }^{1,2,5}$ Atilio Reyes Romero, ${ }^{1}$ Constantinos Neochoritis, ${ }^{3}$ Matthew Groves, ${ }^{1}$ Marco Velasco-Velázquez, ${ }^{2,5}$ Alexander Dömling ${ }^{1,3,4 *}$

$192{ }^{1}$ Department of Drug Design, University of Groningen, The Netherlands

$193{ }^{2}$ Departamento de Farmacología y Unidad Periférica de Investigación en Biomedicina Traslacional, 194 Facultad de Medicina, Universidad Nacional Autónoma de México (UNAM). Ciudad de México, México.

$195{ }^{3}$ Telesis Pharma, Groningen, The Netherlands

$196{ }^{4}$ FORTH, Institute of Molecular Biology and Biotechnology, Crete, Greece

1975 Programa de Doctorado en Ciencias Biomédicas, UNAM. Ciudad de México, México.

198

\section{Supporting information}

\section{Table of contents}

201 Figure S1

202 Virtual screening methodologies 

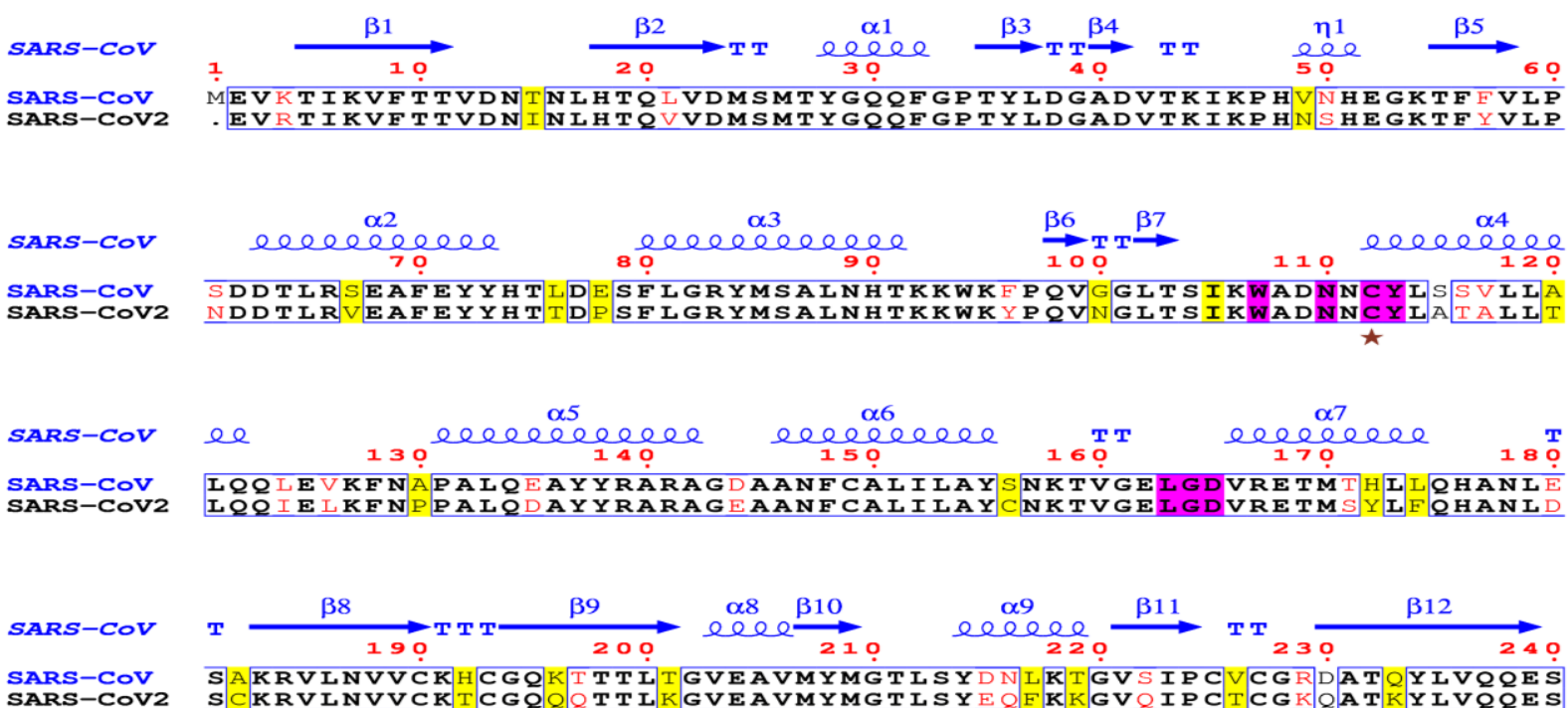

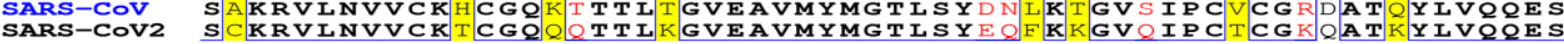

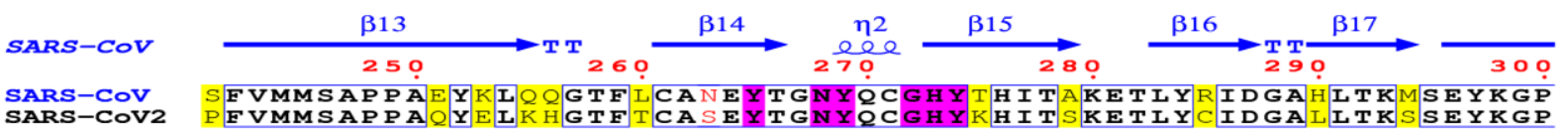

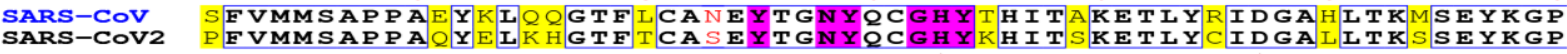

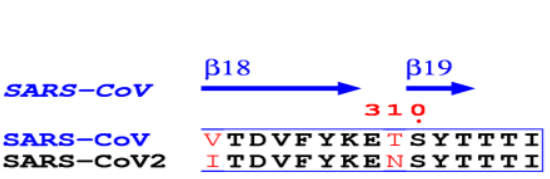

217 Figure S1 Pairwise alignment of SARS-CoV (blue colored, PDB ID: 2FE8) and SARS-CoV-2 (NCBI Reference 218 Sequence: YP_009725299) papain-like proteases (PLpro). ${ }^{1,2}$ The catalytic triad (Cys112, His273 and 219 Asp287) is indicated by a brown stars. ${ }^{3}$ Identical residues are framed in blue, non-identical ones are 220 highlighted in yellow while similar resides colored in red. The residues flagging the binding site are 221 purple boxed. Alignment was done with ClustalW and secondary structures elements were created with 222 EsPript 3.0.4,5

\section{Virtual screening methodologies}

224 GOLD

225 The crystal structures of SARS-CoV-2 3CLpro (PDB ID 6LU7) and SARS-CoV PLpro (PDB ID 2EF8) were used as a receptor and Alogliptin, Anagliptin, Bisegliptin, Denagliptin, Melogliptin, Saxaglipin, Trelagliptin, and Vildagliptin as ligands. Protein were prepared by removing co-crystallized ligand if present, water, and solvent molecules; and adding charges and hydrogens using Chimera $1.14 .{ }^{6}$ Isomeric SMILE codes for ligands were retrieved from PubChem and prepared for docking by setting the absolute stereo flags, adding explicit hydrogens and tautomeric states at pH 7.4. 3D coordinates were generated 
with Standardized 19.20.0 (http://www.chemaxon.com). For the covalent docking, the sulfur atom of the reactive cysteines of the receptors were used as linker to perform the covalent bonding (Cys145 for 3 CLpro and Cys112 for PLpro). For each ligand, 50 runs of genetic algorithm for the conformational search were performed and each pose was evaluated employing the PLP Chemscore scoring function.

\section{CovDock}

The crystal structures of SARS-CoV-2 3CLpro (PDB ID 6LU7) and SARS-CoV PLpro (PDB ID 2EF8) were prepared by assigning bond orders, hydrogens and protonation state at $\mathrm{pH} 7.4$ with Epik and missing side chains/loops were filled with Prime. ${ }^{7-10}$ Water molecules beyond $5.0 \AA$ and DMSO were removed. Finally, the structures were further refined by assigning the optimal $\mathrm{H}$-bonds orientations with PROPKA3 at $\mathrm{pH} 7.4$ and a restrained minimization was then applied with OPLS_2005 until $0.30 \AA ̊$ RMSD convergence. ${ }^{11-13}$ The 3D-structures and the relative tautomers of the gliptins at $\mathrm{pH} 7.4$ from isomeric smiles were respectively generated with Ligprep (Schrödinger Release 2019-4: LigPrep, Schrödinger, LLC, New York, NY, 2020) and Epik, including the stereoflags at the chiral centers. ${ }^{7,8}$ The docking grid was defined in center of coordinates $-14.54 \times 46.05 \times-38.91$ and $20.0 \AA$ of spacing in all directions. Covalent reaction was set as nucleophilic addition to triple bond and docking was run including ligand conformational sampling, rotamer sampling of the protein side chains, and minimization of the pose. A total number of 200 poses were requested employing a cutoff of $2.5 \mathrm{kcal} / \mathrm{mol}$ as a restrain. Subsequently, the best poses were selected based on GlideScore value ( $\mathrm{kcal} / \mathrm{mol}$ unit). ${ }^{14,15}$

For GOLD and CovDock, the cooperativity interaction networks were assessed by Scorpion before and after MAB force field minimization with Moloc. ${ }^{16-18}$ All the figures were rendered with Pymol (The PyMOL Molecular Graphics System, Version 2.3, Schrödinger, LLC).

\section{Consensus score}

For comparison between scores of best poses from GOLD and CovDock for SARS-CoV-2 3CLpro (Table S1, Fig. S2A), SARS-CoV PLpro (Table S2, Fig. S2B), and values from cooperative interaction networks and normalization was applied (Table S3 for 3CLpro and Table S4 for PLpro, Fig. 1A and 1B main text). Negative values from CovDock were converted into positive values by multiplying by -1 , then a 0 to 1 range normalization was done among each method using 1 as the higher value of each group and 0 the lowest. Finally, the consensus score was computed as the sum of each normalized score per compound.

Table S1 Final docking scores obtained by CovDock, GOLD, and Scorpion scores calculated from the best poses of gliptins against SARS-CoV PLpro.

\begin{tabular}{|c|c|c|c|c|}
\hline Compound & CovDock & GOLD & Scorpion_CovDock & Scorpion_GOLD \\
\hline Alogliptin & $-2,89$ & 0 & 7,7 & 1,2 \\
\hline Denagliptin & $-3,63$ & 15,48 & 10,2 & 6,8 \\
\hline Anagliptin & $-2,98$ & 82,52 & 7,6 & 6,5 \\
\hline Bisegliptin & $-2,93$ & 65,67 & 2,9 & 5,1 \\
\hline Melogliptin & $-2,54$ & 73,65 & 10,4 & 7,9 \\
\hline Vildagliptin & $-2,26$ & 72,93 & 7,2 & \\
\hline
\end{tabular}




\begin{tabular}{|c|c|c|c|c|}
\hline Saxagliptin & - & 26,82 & - & 3 \\
\hline Trelagliptin & - & 0 & - & 4,6 \\
\hline
\end{tabular}

261

262 Table S2 Final docking scores obtained by CovDock, GOLD, and Scorpion scores calculated from the best 263 poses of gliptins against SARS-CoV-2 3CLpro.

\begin{tabular}{|c|c|c|c|c|}
\hline Compound & CovDock & GOLD & Scorpion_CovDock & Scorpion_GOLD \\
\hline Alogliptin & $-5,92$ & 89,8825 & 3,4 & 5,0 \\
\hline Vildagliptin & $-6,48$ & 85,007 & 6,7 & 10,1 \\
\hline Denagliptin & $-6,29$ & 90,6431 & 6,9 & 6,3 \\
\hline Anagliptin & $-6,23$ & 94,6538 & 5,7 & 3,3 \\
\hline Melogliptin & -6 & 87,8583 & 5 & 6,2 \\
\hline Bisegliptin & $-5,74$ & 80,1911 & 2,8 & 3,1 \\
\hline Trelagliptin & $-5,61$ & 91,2258 & 5,8 & 7,4 \\
\hline Saxagliptin & $-5,45$ & 92,5049 & 6,9 & \\
\hline
\end{tabular}

264

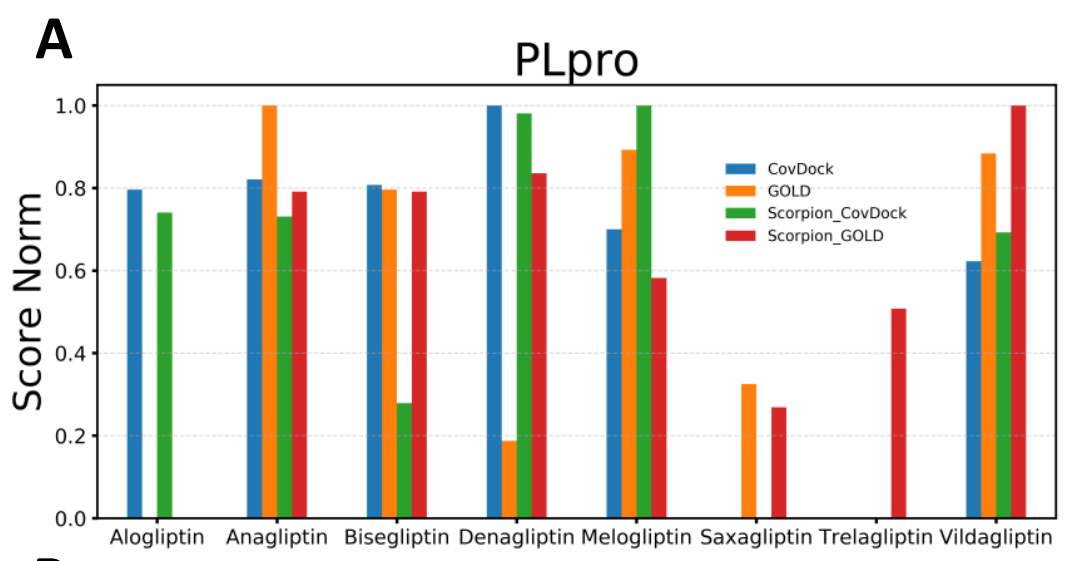

$$
\text { B }
$$

3CLpro

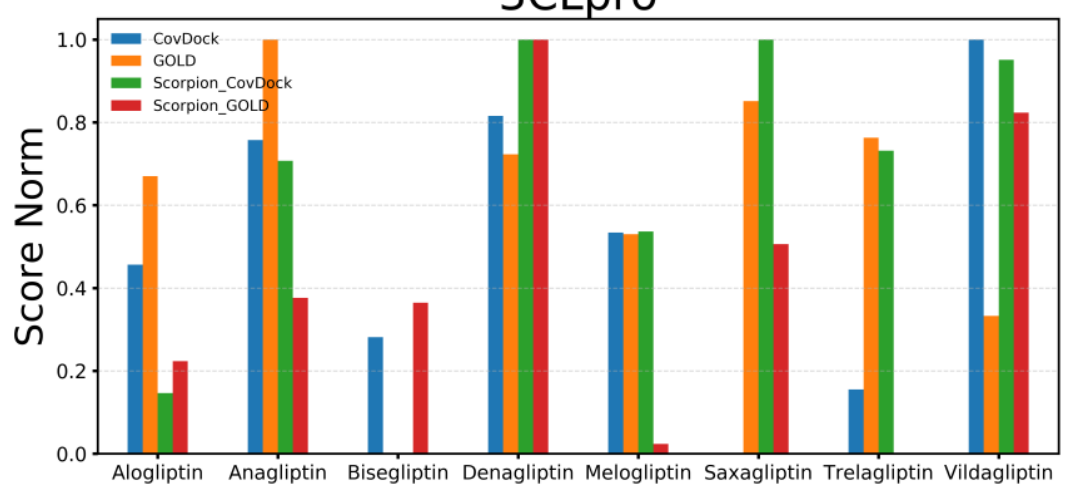


267 Figure S2 Normalized docking scores for papain-like protease (A) and coronavirus main protease (B).

268 Table S3 Normalized and consensus score of gliptins against SARS-CoV PLpro.

\begin{tabular}{|l|c|c|c|c|c|}
\hline Compound & CovDock & GOLD & Scorpion_CovDock & Scorpion_GOLD & Consensus \\
\hline Alogliptin & 0,8 & 0 & 0,74 & 0 & 1,54 \\
\hline Denagliptin & 1 & 0,19 & 0,98 & 0,84 & 3 \\
\hline Anagliptin & 0,82 & 1 & 0,73 & 0,79 & 3,34 \\
\hline Bisegliptin & 0,81 & 0,8 & 0,28 & 0,79 & 2,67 \\
\hline Melogliptin & 0,7 & 0,89 & 1 & 0,58 & 3,17 \\
\hline Vildagliptin & 0,62 & 0,88 & 0,69 & 0,27 & 3,20 \\
\hline Saxagliptin & 0 & 0,33 & 0 & 0,51 & 0,59 \\
\hline Trelagliptin & 0 & 0 & 0 & 0,51 \\
\hline
\end{tabular}

269

270 Table S4 Normalized and consensus score of gliptins against SARS-CoV-2 3CLpro.

\begin{tabular}{|c|c|c|c|c|c|}
\hline Compound & CovDock & GOLD & Scorpion_CovDock & Scorpion_GOLD & Consensus \\
\hline Alogliptin & 0,46 & 0,67 & 0,15 & 0,22 & 1,5 \\
\hline Denagliptin & 0,82 & 0,72 & 1 & 1 & 3,54 \\
\hline Anagliptin & 0,76 & 1 & 0,71 & 0,38 & 2,84 \\
\hline Bisegliptin & 0,28 & 0 & 0 & 0,36 & 0,65 \\
\hline Melogliptin & 0,53 & 0,53 & 0,54 & 0,82 & 1,62 \\
\hline Vildagliptin & 1 & 0,33 & 0,95 & 0,51 & 3,11 \\
\hline Saxagliptin & 0 & 0,85 & 1 & 0 & 2,36 \\
\hline Trelagliptin & 0,16 & 0,76 & 0,73 & 1,65 \\
\hline
\end{tabular}

271 
The best Vinagliptin poses of CovDock (Fig. S3A) and GOLD (Fig. S3B) in 3CLpro form a covalent bond with Cys145. The adamantyl group interacts with His41, Met49, Gly143, Cys145, His163, His164, Met165 side chains through a wide network of Van der Walls interactions (Fig. S3). However, the CovDock pose has one extra hydrogen bond - $\pi$ stacking interaction with the CO backbone moiety of Arg188 while in the GOLD pose was observed an extra Van der Walls interaction with GIn189 side chain and one hydrogen bond/ $\pi$ stacking with NH moiety of Cys112. Both poses show that the imidate group is stabilized by dipolar interaction with carbonyl moiety of Gly143. Additionally, many of these interactions were also observed in the recently published non-covalent designed alpha-ketoamide inhibitor of 3 CLpro. ${ }^{19}$ On the other hand, considerable modification to the backbone of the protease domain of 3 CLpro (residues 10-99) were observed because of the binding of Valdagliptin. These findings together could provide evidence suggesting Valdagliptin, and other reported gliptins as potential inhibitors of SARS-CoV-2 main protease.

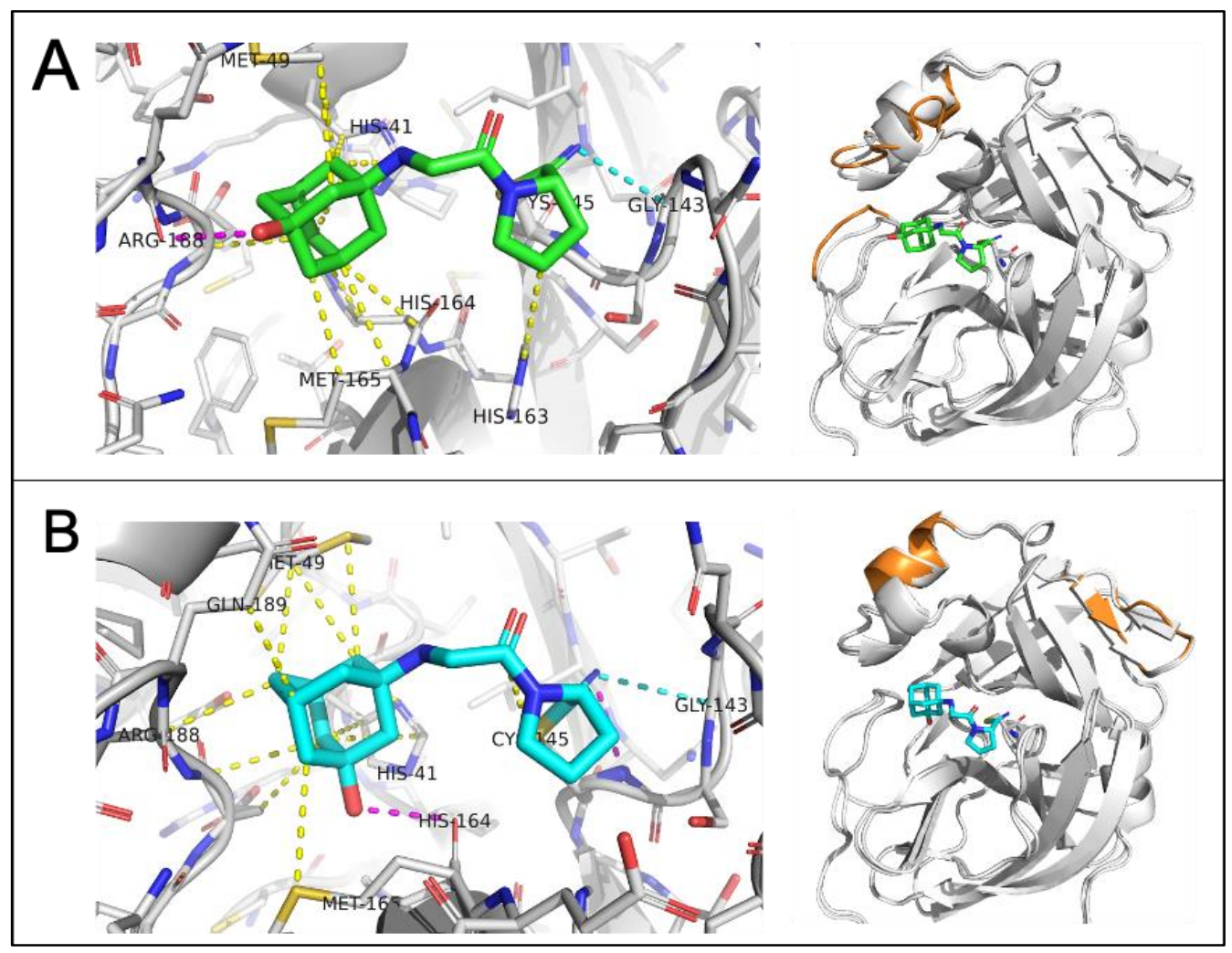

Figure S3 Molecular interactions of Vildagliptin with SARS-CoV-2 3CLpro and induced fit. The figure shows the molecular interactions of best pose of CovDock (A -green sticks-) and GOLD (B -cyan sticks-). Van der Walls interactions are shown as yellow lines, hydrogen bond/ $\pi$ stacking in magenta, and dipolar interactions in cyan. Larger displacements between the apo-3CLpro backbone (white) and Vildagliptin$3 C$ pro complexes after MAB minimizations were observed in the protease domain of $3 \mathrm{CLpro}$ (orange sections). 
307

308

309

310

311

312

313

\section{SARS-CoV PLpro}

Whereas CovDock (Fig. S4A) and GOLD (Fig. S4B), they predicted Van der Walls interactions between the adamantyl group of Vinagliptin and Tyr269, His273, and Tyr274 side chains in addition to the covalent bond between the nitrile and the Cys112. However, in the case of CovDock, the imidate group is stabilized by a hydrogen bond with the indole NH moiety of Trp107 and further Van Der Walls interactions were observed with Leu163, Tyr113, Tyr265 and Asn268 side chains. In the docking pose of GOLD (Fig. S4B), the same imidate stabilization is done by a hydrogen bond/ $\pi$ stacking interaction with $\mathrm{NH}$ moiety of Cys112. Further non-covalent contacts consist in a cation/ $\pi$ stacking interaction between the NH moiety of the ligand and the backbone carbonyl group of Gly164 and the hydroxyl group of Vinagliptin with $\mathrm{OH}$ moiety of Tyr274 side chain. On the other hand, considerable modification to the backbone of the protease domain of PLpro were observed as a consequence of Valdagliptin binding.

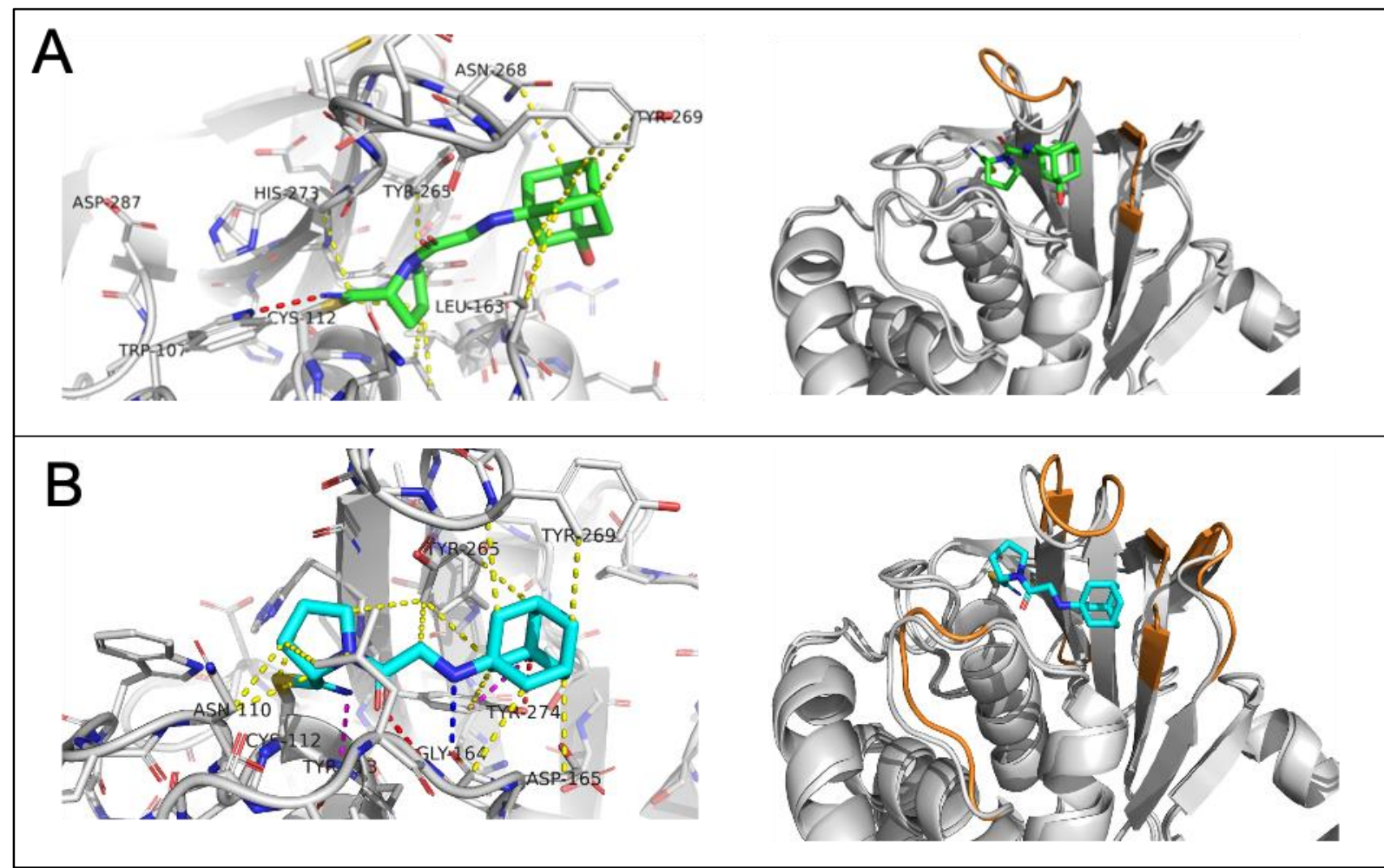

Figure S4 Molecular interactions of Vildagliptin with SARS-CoV PLpro and induced fit. The figure shows the molecular interactions of best pose of CovDock (A, green sticks) and GOLD (B, cyan sticks). Van der Walls interactions are shown as yellow lines, hydrogen bonds in red, and $\pi$-stacking in magenta. Larger displacements (> $2.0 \AA$ ) between the apo-PLpro backbone (white) and Vildagliptin-PLpro complexes after MAB minimizations were observed in the protease domain of PLpro (orange sections). 
The studied gliptins share similar non-covalent interactions with the residues reported for a specific a potent SARS-CoV inhibitor (PDB ID 4OVZ). ${ }^{3}$ (Hydrogen bonds, $\pi-\pi$ stacking, and Vander Walls interactions were found on Tyr269, the formation of a dipolar interaction occurring on CO moiety of Gly164, and one hydrogen bond between the reported inhibitor and Asp165. Several Van der Walls interactions were observed with residues Pro248, Pro249 Thr302, GIn270, Asp165, Asp265, Tyr274, Thr302 where the most relevant non-covalent interactions occurred between the reported inhibitor and Tyr269. Interestingly, our results showed similar interactions between the studied gliptins and Tyr269 and other Plpro protease domain residues (Fig. S4). Additionally, the structural alignment after MAB minimization demonstrated that a indicated an adjusted fit shift on similar regions of the Plpro protease domain in comparison which the modifications driven by the binding of Vildagliptin and other proposed gliptins, particularly in the loop comprised by the Asn268, Tyr269, and Gln270.

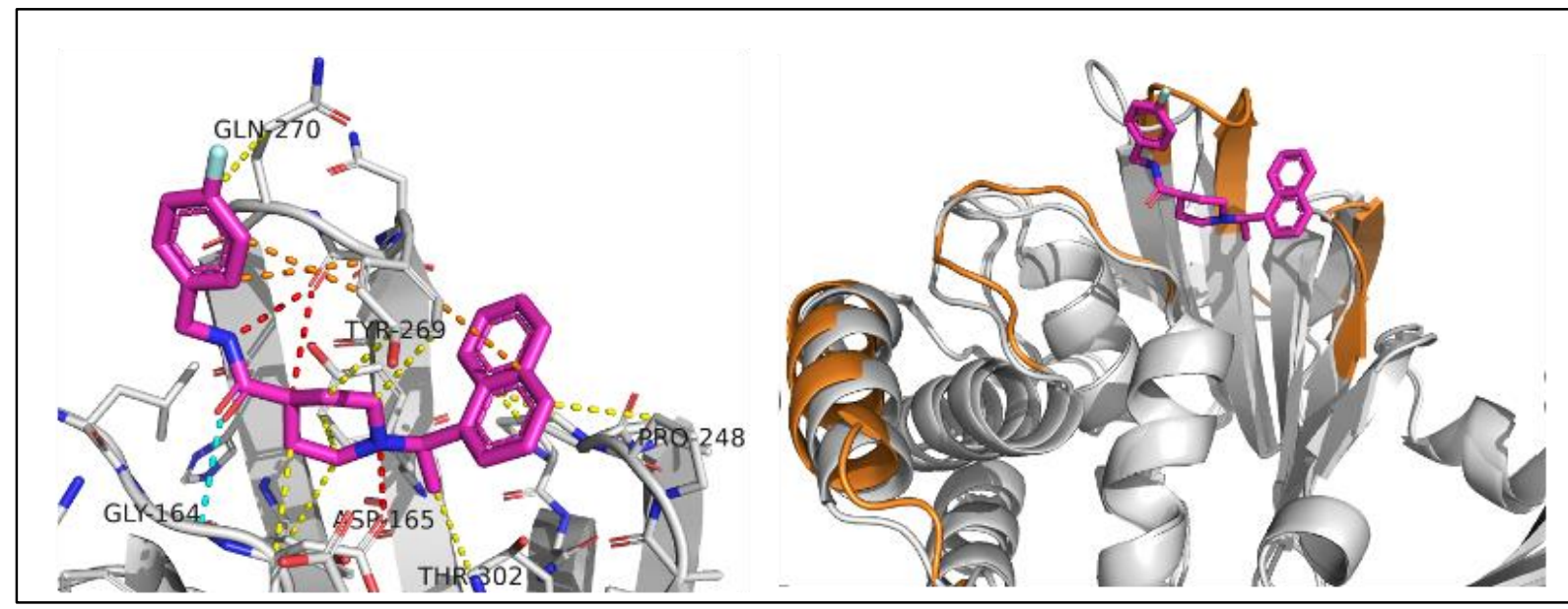

Figure S5 Molecular interactions of reported non covalent inhibitor with SARS-CoV PLpro and induced fit. The ligand is shown as magenta sticks. Van der Walls interactions are shown as yellow lines, hydrogen bonds in red, and $\pi-\pi$ in orange, and dipolar interaction in cyan. Larger displacements (> 2.0 A) between the apo-PLpro backbone (white cartoons) and inhibitor-PLpro complex after MAB force field minimizations were observed in the protease domain of PLpro (orange sections)

\section{References}

1. Ratia K, Saikatendu KS, Santarsiero BD, et al. Severe acute respiratory syndrome coronavirus papainlike protease: Structure of a viral deubiquitinating enzyme. Proc Natl Acad Sci 2006;103(15):571722.

2. Wu F, Zhao S, Yu B, et al. A new coronavirus associated with human respiratory disease in China. Nature 2020;579(7798):265-9.

3. Báez-Santos YM, St John SE, Mesecar AD. The SARS-coronavirus papain-like protease: structure, function and inhibition by designed antiviral compounds. Antiviral Res 2015;115:21-38.

4. Thompson JD, Higgins DG, Gibson TJ. CLUSTAL W: improving the sensitivity of progressive multiple sequence alignment through sequence weighting, position-specific gap penalties and weight matrix choice. Nucleic Acids Res 1994;22(22):4673-80. 
5. Robert X, Gouet P. Deciphering key features in protein structures with the new ENDscript server. Nucleic Acids Res 2014;42(W1):W320-4.

6. Pettersen EF, Goddard TD, Huang CC, et al. UCSF Chimera--a visualization system for exploratory research and analysis. J Comput Chem 2004;25(13):1605-12.

7. Shelley JC, Cholleti A, Frye LL, Greenwood JR, Timlin MR, Uchimaya M. Epik: a software program for pKaprediction and protonation state generation for drug-like molecules. J Comput Aided Mol Des 2007;21(12):681-91.

8. Greenwood JR, Calkins D, Sullivan AP, Shelley JC. Towards the comprehensive, rapid, and accurate prediction of the favorable tautomeric states of drug-like molecules in aqueous solution. J Comput Aided Mol Des 2010;24(6-7):591-60

9. Jacobson MP, Pincus DL, Rapp CS, et al. A hierarchical approach to all-atom protein loop prediction. Proteins 2004;55(2):351-67.

10. Jacobson MP, Friesner RA, Xiang Z, Honig B. On the Role of the Crystal Environment in Determining Protein Side-chain Conformations. J Mol Biol 2002;320(3):597-608.

11. Olsson MHM, Søndergaard CR, Rostkowski M, Jensen JH. PROPKA3: Consistent Treatment of Internal and Surface Residues in Empirical pKa Predictions. J Chem Theory Comput 2011;7(2):525-37.

12. Jorgensen WL, Maxwell DS, Tirado-Rives J. Development and Testing of the OPLS All-Atom Force Field on Conformational Energetics and Properties of Organic Liquids. J Am Chem Soc 1996;118(45):11225-36.

13. Shivakumar D, Williams J, Wu Y, Damm W, Shelley J, Sherman W. Prediction of Absolute Solvation Free Energies using Molecular Dynamics Free Energy Perturbation and the OPLS Force Field. J Chem Theory Comput 2010;6(5):1509-19.

14. Friesner RA, Murphy RB, Repasky MP, et al. Extra Precision Glide: Docking and Scoring Incorporating a Model of Hydrophobic Enclosure for Protein-Ligand Complexes. J Med Chem 2006;49(21):617796.

15. Friesner RA, Banks JL, Murphy RB, et al. Glide: a new approach for rapid, accurate docking and scoring. 1. Method and assessment of docking accuracy. J Med Chem 2004;47(7):1739-49.

16. Kuhn B, Fuchs JE, Reutlinger M, Stahl M, Taylor NR. Rationalizing Tight Ligand Binding through Cooperative Interaction Networks. J Chem Inf Model 2011;51(12):3180-98.

17. Gerber PR, Müller K. MAB, a generally applicable molecular force field for structure modelling in medicinal chemistry. J Comput Aided Mol Des 1995;9(3):251-68.

18. Gerber PR. Topological Pharmacophore Description of Chemical Structures using MAB-Force-FieldDerived Data and Corresponding Similarity Measures. In: Carbó-Dorca R, Gironés X, Mezey PG, editors. Fundamentals of Molecular Similarity. Boston, MA: Springer US; 2001 [cited 2020 Jan 7]. p. 67-81.Available from: https://doi.org/10.1007/978-1-4757-3273-3_5 
378 19. Zhang L, Lin D, Sun X, et al. Crystal structure of SARS-CoV-2 main protease provides a basis for design 